\title{
Minocycline Alleviate A Rat Model Of Gulf War Illness Via Altering Gut Microbiome, Attenuating Neuroinflammation And Enhancing Hippocampal Neurogenesis
}

\section{Liang Liu}

General Hospital of Northern Military Area

\section{Er-Qiang Wang}

Hospital of Fuqing city

\section{Cheng Du}

General Hospital of Northern Military Area Hui-Sheng Chen

General Hospital of Northern Military Area Yan Lv ( $D$ lana323@foxmail.com )

General Hospital of Northern Military Area

\section{Research}

Keywords: Gulf War Illness, Minocycline, Neurogenesis, Gut microbiome, Neuroinflammation

Posted Date: July 28th, 2020

DOI: https://doi.org/10.21203/rs.3.rs-45452/v1

License: (c) (1) This work is licensed under a Creative Commons Attribution 4.0 International License. Read Full License 


\section{Abstract}

Background: Accumulating evidence suggests that deficits in neurogenesis, chronic inflammation and gut microbiome dysregulation contribute to the pathophysiology of Gulf War Illness (GWI). Minocycline has been demonstrated to be a potent neuroprotective agent and could regulate neuroinflammation. The present study intended to investigate whether treatment of minocycline maintain better cognition and mood function in a rat model of GWI and the potential mechanism.

Methods: Rats received 28 days of GWI-related chemical exposure and restraint stress, along with daily minocycline or vehicle treatment. Cognitive and mood function, neuroinflammation, neurogenesis and gut microbiota were detected.

Results: We found that minocycline treatment induced better cognitive and mood function in a GWI rat model, as indicated by open-field test, elevated plus maze test, novel object recognition test and forced swim test. Moreover, minocycline treatment reversed the altered gut microbiome, neuroinflammation and the decreased hippocampal neurogenesis of rats with GWI.

Conclusion: Taken together, our study indicated that minocycline treatment exerts better cognitive and mood function in GWI rat model, which is possible related to gut microbiota remodeling, restrained inflammation and enhanced hippocampal neurogenesis. These results may establish minocycline a potential prophylactic or therapeutic agent for the treatment of GWI.

\section{Introduction}

Gulf War Illness (GWI) is a multi-symptom illness that affects 30\% of veterans from the 1991 Gulf War and is characterized by a spectrum of chronic medically unexplained symptoms including fatigue, reduced ability to concentrate, insomnia, dizziness, functional gastrointestinal disorders, respiratory disorders, chronic pain, headaches, diminished cognitive and mood function ${ }^{1,2}$. The etiology and pathogenesis of GWI is very complex and may be related to veterans' experience in wartime, vaccination, psychological stress, infectious diseases, and exposure to various chemicals, including lean uranium, oil well flame smoke, nerve gas, pyridostigmine bromide (PB) and insecticides ${ }^{3-5}$. Currently, there is increasing evidence suggesting that $\mathrm{PB}$ and pesticides are closely associated with the onset of $\mathrm{GWI}{ }^{6-8}$. These studies also suggested that a majority of veterans with GWI used higher amounts of PB pills and pesticides during the Gulf War. However, to data, there is no therapies available for GWI.

Consistent with the above findings, studies on animal model of GWI also have found that insecticides such as N, N-diethyl-m-toluamide (DEET) and permethrin can cause chronic brain dysfunction, including decreasing adult hippocampal neurogenesis, over activation of neuro-inflammation, damaging the bloodbrain barrier, causing nerve cells death in the dentate gyrus, cingulate cortex, thalamus and hypothalamus ${ }^{9-11}$. Brain dysfunction is typified by depression, anxiety and impaired cognition ${ }^{10,12}$. Meanwhile, it is well-known that adult hippocampal neurogenesis plays a crucial role both in cognitive function and mood 
regulation ${ }^{13}$. Importantly, the impairment of adult hippocampal neurogenesis contributes to several psychiatric disorders, such as GWI, addiction, depression, schizophrenia and autism spectrum disorder 10,14-16. Brain imaging studies of GWI patients suggested that the scores on immediate and delayed verbal and visual retrieval decreased in GWI patients, and the hippocampal volume, especially the hippocampal head, was significantly smaller than that of the healthy civilian ${ }^{17}$. Additionally, accumulating evidence suggests that the level of neuroinflammation in the brain play a role in adult neurogenesis. Over activation of neuroinflammation can adversely affect cognitive and mood function either directly or indirectly by inhibiting hippocampal neurogenesis ${ }^{18,19}$. Meanwhile, over activation of neuroinflammation have been identified in animal models of GWI using exposures to several chemical combinations ${ }^{20}$. For GWI patients, the levels of multiple proinflammatory biomarkers in the blood also increased significantly ${ }^{21}$. More importantly, enhanced neurogenesis, and alleviation of neuroinflammation by curcumin treatment could leads to better cognitive and mood function in GWI rats 22. Taken together, the results of these studies have indicated that over activation of neuroinflammation and impaired neurogenesis in the adult hippocampus might be the key biological processes involved in GWI pathophysiology and may as the potential therapeutic targets for treating GWI.

Minocycline, as a long-acting tetracycline agent, has a profound neuroprotective effect associated with the improvement of cognitive function, mood regulation and neurogenesis during neuroinflammatory conditions. Several studies have reported that minocycline was beneficial in neurological models such as schizophrenia, depression, ischemic stroke, and Parkinson's disease ${ }^{23-26}$. However, the role of minocycline in promoting hippocampal neurogenesis and alleviating of neuroinflammation, including potential cognitive and mood enhancing properties in GWI, remains unclear. We here aimed to evaluate the effects of minocycline treatment on neuroinflammation and neurogenesis in the hippocampus in the rat model of GWI. We correlated these data with the effects of minocycline treatment on diminished cognitive and mood function of GWI rats.

\section{Materials And Methods}

\subsection{Animals and groups}

Adult male Sprague-Dawley rats ( 9 weeks old) were placed in pairs in the plexiglass cage in a temperature-controlled room $\left(23-25^{\circ} \mathrm{C}\right)$ with humidity $50-60 \%$, a 12-h light/12-h dark cycle and ad libitum access to food and water. The experiments were approved by the Animal Care and Use Committee of the General Hospital of Northern Theater Command and was in consistence with the principles outlined in the National Institutes of Health Guide. All efforts were made to minimize the number of animals used at each step. Rats were randomly divided into the following five groups: Control group, GWI group, and GWI + Mino group.

\subsection{Exposure of rats to PB, Permethrin and restrained-stress}


The pyridostigmine bromide (PB) was purchased from Sigma (Burlington, MA, USA) and was administered by oral gavage at a dose of $1.3 \mathrm{mg} / \mathrm{kg}$ for $28 \mathrm{~d}$ (in $500 \mu \mathrm{L}$ water). The rats exposed to chemicals DEET (in $200 \mu \mathrm{L} 70 \%$ alcohol, $40 \mathrm{mg} / \mathrm{kg}$ ) and PM (in $200 \mu \mathrm{L} \mathrm{70 \%} \mathrm{alcohol,} 0.13 \mathrm{mg} / \mathrm{kg}$ ) over shaved skin areas located on the back of the neck and the upper thoracic region for 28 consecutive days. The doses of PB, PM, and DEET were chosen according to previous reports. ${ }^{10}$ Moreover, 5 min of daily restraint stress was conducted for 28 days by a rat restrainer, as previously reported. ${ }^{27}$

\subsection{Administration of minocycline}

As Figure 1 show that rats received PB, Permethrin and restrained-stress exposure and with normal saline intraperitoneal or minocycline (Sigma) dissolved in sterile saline, at dose of $40 \mathrm{mg} / \mathrm{kg} / \mathrm{day}$ (minocycline) by intraperitoneal administration for 28 days.

\subsection{BrdU labeling}

Bromodeoxyuridine (BrdU; Sigma-Aldrich) was dissolved in sterile saline. In order to assess cell proliferation in the DG, rats were received BrdU at $50 \mathrm{mg} / \mathrm{kg}$ body weight dose intraperitoneally, for a total of 3 injections in 3 days, and were then sacrificed $24 \mathrm{~h}$ after the last BrdU injection. In order to analyze neurogenesis in the DG, mice were sacrificed 28 days after the first BrdU injection, and BrdU positive cells as well as BrdU positive cells colabeled with doublecortin (DCX) were quantified.

\subsection{Behavioral experiments}

After GWI model established and levofloxacin, minocycline treatment, the behavioral tests were performed. Before each test, rats were acclimatized to the experimental room for at least $30 \mathrm{~min}$. The behavioral tests were performed in the following order: open field test, the elevated plus maze test, novel object recognition test and forced swim test.

\subsubsection{Open field test}

The open-field test was performed according to previous methods ${ }^{28}$, using a video camera secured to the top of the apparatus and analyzed using Ethovision 11.0 (Noldus). Rats were placed in the center of the open-field box and activity was recorded for a period of $10 \mathrm{~min}$. The distance and duration of time in the central area were recorded. After each test, the apparatus was wiped with alcohol and water between tests to eliminate any smell.

\subsubsection{The elevated plus maze test}

The elevated plus maze test was performed to measure anxiety-like behaviors, consisted of two open arms and two closed arms. According to a previous report ${ }^{27}$, the rat was initially placed in the center area facing an open arm. The duration of time for the rat to enter any of the 4 arms was recorded when all 4 paws crossed from the central region into an arm during the 10 min testing period. The duration of time 
spent in the open arms and the number of total arm entries were used for calculation of the extent of anxiety-like behavior in each rat.

\subsubsection{Novel object recognition test}

The novel object recognition test paradigm consisted of mainly two phases. In the adaptive phase, two identical cylindrical objects (A object) are placed on both sides of the test box, place the rat in the box for 3 minutes, and let the rat rest for 15 minutes. During the test phase, one of the cylindrical objects was replaced by a cube box (B object). The length of the bottom of the cube box is the same as the previous cylindrical object. The diameter of the cylindrical box is the same, then put the rat into the test box and let it explore for 3 minutes. The duration of the rat explores new object (B object) and old object (A object) were recorded and analyzed by Ethovision 11.0 (Noldus). As the distance between the tip of nose and object is less than $2 \mathrm{~cm}$, definite as exploration time. Exploration preference index $=$ time for mice to explore new objects $(B) /$ total time for mice to explore all objects $(A+B) * 100 \%$.

\subsubsection{Forced swim test}

Forced swim test is widely used for measurement of depression-like behavior in rodents ${ }^{29}$. Briefly, mice were individually placed for $6 \mathrm{~min}$ in beakers (height: $46 \mathrm{~cm}$, diameter: $30 \mathrm{~cm}$ ) containing $30 \mathrm{~cm}$ height of water $\left(25^{\circ} \mathrm{C}\right)$ for $6 \mathrm{~min}$. During this time the rat adopts an immobile posture to keep its head above water, characterized by motionless floating and the cessation of struggling. The latency to adopt this posture and total time spent immobile during the last 4 min of the 6 min test were recorded. Increased duration of immobility indicated a state of helplessness.

\subsection{Immunohistochemistry and immuno-fluorescence}

Immunohistochemistry was conducted according to previous method ${ }^{28}$. Briefly, rats were deeply anesthetized with an overdose of isoflurane and transcardially perfused with $0.01 \mathrm{M}$ phosphatebuffered saline (PBS, $\mathrm{pH} 7.4$ ) for 5-10 min, followed by $4 \%$ paraformaldehyde in $0.1 \mathrm{M}$ phosphate buffer (PBS, $\mathrm{pH}$ 7.4) for $15-20 \mathrm{~min}$. Whole brains were removed and post-fixed in the same fixative for 3-4 $\mathrm{d}$ at $4{ }^{\circ} \mathrm{C}$ followed by $30 \%$ sucrose treatment at $4{ }^{\circ} \mathrm{C}$. Serial coronal brain sections ( 5 or $40 \mu \mathrm{m}$ in thickness) were collected. The sections were first washed in $0.01 \mathrm{M}$ PBS to remove the cryoprotectant solution, and then incubated in $3 \% \mathrm{H}_{2} \mathrm{O}_{2}$ for 30 min at room temperature to quench endogenous peroxidase, followed by washing with $0.01 \mathrm{M}$ PBS. The sections were then incubated with primary antibodies for $12 \mathrm{~h}$ at $4{ }^{\circ} \mathrm{C}$, including rabbit anti-NeuN (1:1000, BD, San Jose, CA) or goat anti-DCX (1:200, Santa Cruz Biotechnology, CA, USA). For BrdU staining, all the sections were pretreated with $2 \mathrm{~N} \mathrm{HCl}$ for $1 \mathrm{~h}$ at $37^{\circ} \mathrm{C}$ to denature the DNA followed by $0.1 \mathrm{M}$ borax $(\mathrm{pH} 8.5)$ treatment for $10 \mathrm{~min}$ to neutralize before the regular immunostaining procedure. After washing, the sections were incubated with biotinylated secondary antibody (1:200, Dako, Glostrup, Denmark) $\left(2 \mathrm{~h}, 37^{\circ} \mathrm{C}\right)$, followed by the avidin-biotin complex (Dako). Finally, sections were analyzed under a Lycra microscope.

\subsection{Quantitative RT-PCR}


Total RNA was extracted using the TRIZOL reagent (Sigma Aldrich) according to the manufacturer's instructions. And then cDNA was generated using the PrimeScript ${ }^{\circledR} R T$ Reagent Kit (Takara Bio Inc) and qPCR was performed in triplicate using 96 wells PCR plates and YBR Green qPCR Mix (Takara Bio Inc., Shiga, Japan) with a CFX96 Real-time PCR System (Bio-Rad) according to manufacturer's instructions. Cq values were normalized to GAPDH and were calculated using the $2-\Delta \Delta C(\mathrm{t})$ method as described previously ${ }^{30}$.

\subsection{ELISA}

The concentration of rat IL-10 (Wuhan USCN) and IL-17 (Wuhan USCN) in the hippocampus and serum were measured by their respective ELISA kits according to the manufacturers' instructions. The results are expressed as picograms milligram $(\mathrm{pg} / \mathrm{mg})$ or picograms permilliliter $(\mathrm{pg} / \mathrm{ml})$.

\subsection{Western blotting}

The whole hippocampal tissues were homogenized in the RIPA buffer (Beyotime Institute of Biotechnology) and spun down at 13,000 rpm for $10 \mathrm{~min}$, and then supernatants were collected. The protein concentration was determined with a BCA kit (Beyotime Institute of Biotechnology). The rest hippocampal homogenized lysates were performed at $100^{\circ} \mathrm{C}$ for $10 \mathrm{~min}$ followed. The separated proteins were transferred onto a $12 \%$ SDS-polyacrylamide gel at $80 \mathrm{~V}$ for $120 \mathrm{~min}$, then transferred to PVDF membranes. Blotting membranes were blocked in solution ( $5 \%$ nonfat dried milk powder dissolved in TBST buffer at room temperature for $3 \mathrm{~h}$, and then washed three times. Next, membranes were incubated overnight at $4{ }^{\circ} \mathrm{C}$ with primary antibodies against TLR4 (1:1000, Santa Cruz Biotechnology), NF-KB p65 (1:2000, BD Biosciences), $\beta$-actin (1:2000, Cell CWBIO). The membranes were washed and incubated for 2 $\mathrm{h}$ with horseradish peroxidase-conjugated secondary antibody (1:1000, Santa Cruz Biotechnology). The bands were scanned and analyzed using a chemiluminescence system (Bio-RAD Laboratories Inc.).

\subsection{Microbiome analysis}

Fecal pellets and luminal contents were collected from the animals of each group after sacrifice, and then bacterial DNA from cecal contents was extracted using a DNeasy PowerSoil Kit (Qiagen) according to the manufacturer's instructions. 16s rRNA gene sequencing of fecal DNA samples was performed by NovaSeq sequencing platform. The V3-V4 region was amplified and sequencing was done using an Illumina HiSeq sequencing platform. Sequences from all samples were processed using QIIME (Knight and Caporaso labs) and then assigned by the RDP classifier (Michigan State University) against the Greengenes database.

\subsection{Statistical analyses}

All data are presented as the mean \pm SEM and analyzed using SPSS 20.0 software (SPSS Inc., Chicago, IL, USA). Data analyses were performed using one-way ANOVAs and significant effects were evaluated with Tukey's post hoc tests. In the 16S rRNA gene sequencing analysis, a repeated-measure ANOVA was 
used to analyze the a-diversity of the microbiota to assess treatment-by-sequence interactions. Statistically significance was set at $p<0.05$.

\section{Results}

\subsection{Minocycline treatment alleviated neurobehavioral deficits in a GWI rat model}

A timeline with the experimental design was shown in Figure 1A. As previous studies reported that animals with GWI exhibited increased levels of depressive- and anxiety-like behaviors ${ }^{10}$. Thus, we examined a cohort of rats using the open field test and the elevated plus maze test to examine locomotor activity and anxiety-related behaviors. We found that minocycline treatment reverses decreased time in the central area (Figure 1B) for GWI rats and decreased distance in the central area (Figure 1C) in the open field test. Meanwhile, the results from the elevated plus maze test showed that rats in GWI + Mino group displayed higher level in the percentage of time spent in open-arms (Figure 1D) and percentage of open arm entries (Figure 1E), compared with rats in GWI group. Moreover, as previously reported that decreased immobility time in the forced swim test is the index of antidepressant-like efficacy. We revealed that GWI rats showed increased immobility in the forced swim test, compared with control rats. While minocycline treatment reversed this effect significantly in GWI rats (Figure 1F). These results indicated that minocycline treatment decreases depressive- and anxiety-like behaviors in GWI rats.

To test whether minocycline corrected recognition memory deficit in GWI rats, we performed the novel object recognition test. The results showed that GWI rats had a deficit in declarative recognition memory, whereas control rats exhibited a significant preference for the novel object. GWI rats with minocycline treatment for 28 days significantly improved recognition memory compared to that of the vehicleadministered GWI group (Figure 1G). Meanwhile, the total exploratory time of all objects (Figure 1H) or the total distance moved in the box (Figure 11) were comparable across the three groups, indicating that the results are not influenced by potential changes in overall activity or lack of motivation. Thus, these findings implied that minocycline treated GWI rats exhibited better recognition memory.

\subsection{Minocycline treatment enhanced hippocampal neurogenesis in GWI rats}

The hippocampal pathology of GWI typified by decreased neurogenesis, while enhanced hippocampus neurogenesis could alleviate GWI-related behaviors ${ }^{10,22}$. Therefore, we analyzed the proliferating cell population in the SGZ-GCL using the BrdU incorporation assay, which demonstrated that a reduced density of BrdU+ cells in GWI rats compared to control group. While minocycline treatment rescued the decreased BrdU+ cells in the SGZ-GCL of GWI rats significantly (Figure 2A-B). Furthermore, we analyzed the percentages of BrdU+ cells expressing DCX in the SGZ-GCL at 14 days following BrdU injections. We found that no difference in the percentages of BrdU+ cells expressing DCX between the three groups (Figure 2C-D). While, the DCX+ cells decreased significantly in GWI rats compared to control rats, which indicated that the number of newly born immature neurons were diminished in the GWI rats, while this effect was blocked by minocycline treatment (Figure 2E). Besides, we further analyzed the percentages of 
BrdU+ cells expressing NeuN in the SGZ-GCL at 28 days following BrdU injections. The data showed that no difference in the neuronal fate-choice decision by newly born cells between the three groups (Figure $2 \mathrm{~F}-\mathrm{G})$.

\subsection{Minocycline treatment attenuated chronic systemic and brain inflammation in GWI rats}

Previous studies revealed that exposure to Gulf war illness-related chemicals resulted in the immune system dysfunction ${ }^{21}$. Our results showed that the concentration of IL-17 was elevated both in serum and hippocampus of GWI rats and this increase was attenuated by minocycline treatment (Figure 3A, B). Moreover, the level of the anti-inflammatory marker of IL-10 in serum and hippocampus of GWI rats was significantly higher than the rats in the control group, but minocycline treatment increased the level of IL10 significantly for GWI rats (Figure 3C, D).

Furthermore, as previous studies reported that the TLR4/NF-KB signaling pathway is involved in the process of neuroinflammation ${ }^{31}$. Thus, to reveal the mechanism of minocycline treatment for regulation of neuroinflammation, we detected the expression of TLR4 and NF-KB p65 signaling pathway in hippocampus of rats after minocycline treatments by quantitative RT-PCR and western blotting (WB). Our results showed that the expression of TLR4 and NF-KB p65 were elevated significantly in GWI rats and these increases were attenuated by minocycline treatment (Figure 3E-H).

\subsection{The effects of minocycline treatment on microbial diversity and the microbial community in GWI rats}

As exposure of gulf war chemicals and the resultant chronic stress could alter the gut microbiome in the GWI animal model, we analyzed the composition of rat gut microbiota in stool samples by nextgeneration sequencing using V3-V4 hyper-variable 16S rRNA genomic region. Results showed that gulf war chemicals and the resultant chronic stress altered bacterial alpha diversity in the intestinal lumen significantly. Compared with the Control group, the GWI group had significantly increased commensal richness and diversity, as confirmed by the increased ACE index (Figure 4A) and Shannon diversity (Figure $4 B)$. The ACE index and Shannon diversity was decreased in the GWI+Mino group compared with the GWI group (Figure 4A-B). We also evaluated the dissimilarity between cecum bacterial communities among the groups by using principal coordinate analysis (PCOA) of the weighted UniFrac distance. The results showed that minocycline treatment reshaped the microbiota of rats with GWI (Figure 4C). Moreover, we found that there were significant differences in the composition of gut bacteria both at the class and family levels between the groups (Figure 4D-E). The class Bacilli, Erysipelotrichia and Deltaproteobacteria were underrepresented and Bacteroidia, Gammaproteobacteria, Spirochaetia and Mollicutes were overrepresented in GWI group compared with Control group, while minocycline treatment rescued these effects for rats with GWI (Figure 4D). In addition, the abundance of the partial families that increased in GWI group, including Ruminococcaceae, Succinivibrionaceae, Lachnospiraceae, Prevotellaceae and Muribaculaceae, but Lactobacillaceae, Peptostreptococcaceae and Erysipelotrichaceae decreased. These changes in GWI rats also were rescued by minocycline treatment (Figure 4E). 


\section{Discussion}

In this study, we provided new evidence that minocycline treatment alleviated impaired cognitive and mood function in a rat model of GWI. Specifically, we observed that these functional benefits may are paralleled by changes in neuroinflammation, hippocampal neurogenesis and gut microbiome. To the best of our knowledge, our report is the first to present comprehensive evidence that minocycline treatment is efficacious to alleviate GWI-related behaviors.

It has been shown that impairments in mood and cognitive function are the key brain abnormalities observed both in veterans with GWI and animal models ${ }^{10,32}$. The hippocampal pathology of GWI mainly include decreased neurogenesis, partial loss of principal neurons, mild inflammation and reduced hippocampal volume ${ }^{10,33}$. These changes in hippocampus showed an association with mood and cognitive dysfunction in a model of GWI. There are credible evidences to support the hypothesis that adult hippocampal neurogenesis in the hippocampus contributes to mood regulation. Especially, in some rodent models of depression and patients, reduced the size of hippocampus may be related to decreased neurogenesis and mature neuronal cell loss ${ }^{34}$. While the therapeutic action of antidepressants mainly depends on enhancing hippocampal neurogenesis and ablation of hippocampal neurogenesis impairs antidepressant efficacy ${ }^{35,36}$. Moreover, previous research has indicated that enhanced neurogenesis leads to better cognitive and mood function in a model of $\mathrm{GWI}{ }^{10}$. Consistent with these findings, we found minocycline treatment alleviated depressive- and anxiety-like behaviors and recognition memory in GWI rats. Meanwhile, substantial decreases in hippocampal neurogenesis were found in a model of GWI, while enhance hippocampal neurogenesis has been observed after minocycline treatment in rats with GWI. Remarkably, minocycline also showed the ability of enhance neurogenesis in others animal models, such as depression, schizophrenia and aging ${ }^{37-39}$. Thus, it is likely that the effects of minocycline on GWI-related behaviors may involve in enhance hippocampal neurogenesis.

Accumulating evidences suggest that chronic inflammation play a crucial role in regulating hippocampal neurogenesis ${ }^{40}$. Especially, neuroinflammation is one of the most essential factors in the progression of GWI. As previous reports, excessive neuroinflammation presented in veterans suffering from GWI and also observed in animal model of GWI ${ }^{21,41}$. Part of serum proteins associated with inflammation were significantly increased in veterans suffering from GWI, including interleukin 6, C-reactive protein, matrix metalloproteinase-9 (MMP-9) and matrix metalloproteinase-2 (MMP-2) ${ }^{42,43}$. In animal model of GWI, suppression of the inflammatory responses showed a neuroprotective effect, improved neurogenesis, and better cognitive and mood function ${ }^{44}$. Previous research has indicated that excessive neuroinflammation showed negative effect on neurogenesis in the hippocampus ${ }^{18}$. Interestingly, in the present study, we found minocycline treatment suppressed neuroinflammation in association with enhanced neurogenesis, better cognitive and mood function in animal models of GWI, indicating a linkage between these processes. Moreover, TLR4/NF-KB pathway in the most important regulators of inflammatory cascades in neuroinflammation. It has been reported that activation of the TLR4/NF-KB pathway leads to acute activation of microglia, which results in the release of pro-inflammatory cytokines. In our study, we have 
observed that minocycline treatment can reverse increased levels of TLR4 and NF-KB in hippocampus of rats with GWI. Consistently, previous studies also have revealed the anti-inflammatory effect of minocycline in other models of neurodegenerative and neuroinflammatory diseases, including Alzheimer's disease, depression, intracerebral hemorrhage and etc. ${ }^{45}$ Thus, our results suggest that minocycline show an anti-inflammatory effect by interrupting of the TLR4/NF-KB signaling pathway.

Studies have shown that stress can change the composition of gut microbiota, and disruptions of the gut microbiota contribute to the changes of systemic inflammation, behaviors, and cognitive function ${ }^{46,47}$. In detail, previous studies have revealed that disruptions of the gut microbiota can decrease the number of regulatory $T$ cells (Treg cells), and leading to an increase in interleukin (IL)-17-positive $\gamma \delta T$ cells through altered dendritic cell activity in small intestine ${ }^{48}$. Moreover, IL-10 and IL-17 are required for the neuroprotection afforded by intestinal dysbiosis. Further, increasing the number of Treg cells in the intestine can reduce the migration of intestinal of effector $T$ cells from the gut to the leptomeninges, thereby reducing the inflammation of brain. Firas et al. have revealed that several gram negative bacterial genera were increased in an established rodent model of GWI ${ }^{49}$. Gut dysbiosis with simultaneous leaky gut and systemic endotoxemia-induced TLR4 activation contributes to GWI chemical-induced neuroinflammation and gastrointestinal disturbances. Evidence found in our study showed that a direct relationship of gulf war chemical exposure and altered gut microbiota diversity with changes in the Phylum and family levels.

\section{Conclusion}

In summary, our study provided the first evidence that minocycline treatment alleviated impaired cognitive and mood function via reversing gut dysbiosis, the dysregulation of the neuroinflammation and restoring hippocampal neurogenesis in a rat model of GWI. Our study suggested that close attention should be paid to the link between GWI and dysbiosis. Our findings provide evidence that minocycline can be a novel strategy that are efficacious for repairing gut dysbiosis, suppressing inflammation and enhancing neurogenesis may be helpful for improving mood and cognitive function in veterans with GWI.

\section{Declarations}

\section{Ethical Approval and Consent to participate}

Not applicable

\section{Consent for publication}

Not applicable.

\section{Availability of data and materials}

Not applicable. 


\section{Competing interests}

The authors declare that they have no competing interests.

\section{Funding}

The work was supported by grants from the Military Logistics Research Projects of China (No. CLB18J048).

\section{Authors' contributions}

Liang Liu and Yan Lv performed animal study and wrote the main body of the manuscript. Hui-Sheng Chen provided critical writing in the revised manuscript. Cheng Du and Yan Lv designed and instructed the writing of the manuscript. All authors read and approved the final manuscript.

\section{Acknowledgements}

Not applicable.

\section{Authors' information}

Liang Liu: 18580763671@163.com

Er-Qiang Wang: 270599282@qq.com

Cheng Du: 767920128@qq.com

Hui-Sheng Chen: chszh@aliyun.com

Yan Lv: lana323@foxmail.com

\section{References}

1. Golomb BA. Acetylcholinesterase inhibitors and Gulf War illnesses. Proc Natl Acad Sci US A. 2008;105(11):4295-4300.

2. Zhou Q, Verne ML, Zhang B, Verne GN. Evidence for Somatic Hypersensitivity in Veterans With Gulf War Illness and Gastrointestinal Symptoms. Clin J Pain. 2018;34(10):944-949.

3. Haley RW, Kurt TL. Self-reported exposure to neurotoxic chemical combinations in the Gulf War. A cross-sectional epidemiologic study. JAMA. 1997;277(3):231-237.

4. Macht VA, Woodruff JL, Maissy ES, et al. Pyridostigmine bromide and stress interact to impact immune function, cholinergic neurochemistry and behavior in a rat model of Gulf War Illness. Brain Behav Immun. 2019;80:384-393.

5. Steele L, Sastre A, Gerkovich MM, Cook MR. Complex factors in the etiology of Gulf War illness: wartime exposures and risk factors in veteran subgroups. Environ Health Perspect. 2012;120(1):112- 
118.

6. Macht VA, Woodruff JL, Grillo CA, Wood CS, Wilson MA, Reagan LP. Pathophysiology in a model of Gulf War Illness: Contributions of pyridostigmine bromide and stress. Psychoneuroendocrinology. 2018;96:195-202.

7. Moss JI. Gulf War Illnesses are autoimmune conditions caused by the direct effect of the nerve gas prophylaxis drug (pyridostigmine bromide) on anergic immune system lymphocytes. Med Hypotheses. 2019;132:109373.

8. White RF, Steele L, O'Callaghan JP, et al. Recent research on Gulf War illness and other health problems in veterans of the 1991 Gulf War: Effects of toxicant exposures during deployment. Cortex. 2016;74:449-475.

9. Joshi U, Pearson A, Evans JE, et al. A permethrin metabolite is associated with adaptive immune responses in Gulf War Illness. Brain Behav Immun. 2019;81:545-559.

10. Parihar VK, Hattiangady B, Shuai B, Shetty AK. Mood and memory deficits in a model of Gulf War illness are linked with reduced neurogenesis, partial neuron loss, and mild inflammation in the hippocampus. Neuropsychopharmacology. 2013;38(12):2348-2362.

11. Amourette C, Lamproglou I, Barbier L, et al. Gulf War illness: Effects of repeated stress and pyridostigmine treatment on blood-brain barrier permeability and cholinesterase activity in rat brain. Behav Brain Res. 2009;203(2):207-214.

12. Ford JD, Campbell KA, Storzbach D, Binder LM, Anger WK, Rohlman DS. Posttraumatic stress symptomatology is associated with unexplained illness attributed to Persian Gulf War military service. Psychosom Med. 2001;63(5):842-849.

13. Snyder JS, Soumier A, Brewer M, Pickel J, Cameron HA. Adult hippocampal neurogenesis buffers stress responses and depressive behaviour. Nature. 2011;476(7361):458-461.

14. Chambers RA. Adult hippocampal neurogenesis in the pathogenesis of addiction and dual diagnosis disorders. Drug Alcohol Depend. 2013;130(1-3):1-12.

15. Sahay A, Hen R. Adult hippocampal neurogenesis in depression. Nat Neurosci. 2007;10(9):11101115.

16. Kang E, Wen Z, Song H, Christian KM, Ming GL. Adult Neurogenesis and Psychiatric Disorders. Cold Spring Harb Perspect Biol. 2016;8(9).

17. O'Donovan A, Chao LL, Paulson J, et al. Altered inflammatory activity associated with reduced hippocampal volume and more severe posttraumatic stress symptoms in Gulf War veterans. Psychoneuroendocrinology. 2015;51:557-566.

18. de Miranda AS, Zhang CJ, Katsumoto A, Teixeira AL. Hippocampal adult neurogenesis: Does the immune system matter? J Neurol Sci. 2017;372:482-495.

19. Ryan SM, Nolan YM. Neuroinflammation negatively affects adult hippocampal neurogenesis and cognition: can exercise compensate? Neurosci Biobehav Rev. 2016;61:121-131. 
20. Michalovicz LT, Locker AR, Kelly KA, et al. Corticosterone and pyridostigmine/DEET exposure attenuate peripheral cytokine expression: Supporting a dominant role for neuroinflammation in a mouse model of Gulf War Illness. Neurotoxicology. 2019;70:26-32.

21. Madhu LN, Attaluri S, Kodali M, et al. Neuroinflammation in Gulf War Illness is linked with HMGB1 and complement activation, which can be discerned from brain-derived extracellular vesicles in the blood. Brain Behav Immun. 2019;81:430-443.

22. Kodali M, Hattiangady B, Shetty GA, Bates A, Shuai B, Shetty AK. Curcumin treatment leads to better cognitive and mood function in a model of Gulf War Illness with enhanced neurogenesis, and alleviation of inflammation and mitochondrial dysfunction in the hippocampus. Brain Behav Immun. 2018;69:499-514.

23. Deakin B, Suckling J, Barnes TRE, et al. The benefit of minocycline on negative symptoms of schizophrenia in patients with recent-onset psychosis (BeneMin): a randomised, double-blind, placebo-controlled trial. Lancet Psychiatry. 2018;5(11):885-894.

24. Zhang C, Zhang YP, Li YY, et al. Minocycline ameliorates depressive behaviors and neuro-immune dysfunction induced by chronic unpredictable mild stress in the rat. Behav Brain Res. 2019;356:348357.

25. Yew WP, Djukic ND, Jayaseelan JSP, et al. Early treatment with minocycline following stroke in rats improves functional recovery and differentially modifies responses of peri-infarct microglia and astrocytes. J Neuroinflammation. 2019;16(1):6.

26. Choi Y, Kim HS, Shin KY, et al. Minocycline attenuates neuronal cell death and improves cognitive impairment in Alzheimer's disease models. Neuropsychopharmacology. 2007;32(11):2393-2404.

27. Parihar VK, Hattiangady B, Kuruba R, Shuai B, Shetty AK. Predictable chronic mild stress improves mood, hippocampal neurogenesis and memory. Mol Psychiatry. 2011;16(2):171-183.

28. Duan CM, Zhang JR, Wan TF, Wang Y, Chen HS, Liu L. SRT2104 attenuates chronic unpredictable mild stress-induced depressive-like behaviors and imbalance between microglial M1 and M2 phenotypes in the mice. Behav Brain Res. 2020;378:112296.

29. Yankelevitch-Yahav R, Franko M, Huly A, Doron R. The forced swim test as a model of depressive-like behavior. J Vis Exp. 2015(97).

30. Livak KJ, Schmittgen TD. Analysis of relative gene expression data using real-time quantitative PCR and the 2(-Delta Delta C(T)) Method. Methods. 2001;25(4):402-408.

31. Chen J, Wang Z, Zheng Z, et al. Neuron and microglia/macrophage-derived FGF10 activate neuronal FGFR2/PI3K/Akt signaling and inhibit microglia/macrophages TLR4/NF-kappaB-dependent neuroinflammation to improve functional recovery after spinal cord injury. Cell Death Dis. 2017;8(10):e3090.

32. Mori DL, Sogg S, Guarino P, et al. Predictors of exercise compliance in individuals with Gulf War veterans illnesses: Department of Veterans Affairs Cooperative Study 470. Mil Med. 2006;171(9):917-923. 
33. Li X, Spence JS, Buhner DM, et al. Hippocampal dysfunction in Gulf War veterans: investigation with ASL perfusion MR imaging and physostigmine challenge. Radiology. 2011;261(1):218-225.

34. Wang Z, Neylan TC, Mueller SG, et al. Magnetic resonance imaging of hippocampal subfields in posttraumatic stress disorder. Arch Gen Psychiatry. 2010;67(3):296-303.

35. Santarelli L, Saxe M, Gross C, et al. Requirement of hippocampal neurogenesis for the behavioral effects of antidepressants. Science. 2003;301(5634):805-809.

36. Hill AS, Sahay A, Hen R. Increasing Adult Hippocampal Neurogenesis is Sufficient to Reduce Anxiety and Depression-Like Behaviors. Neuropsychopharmacology. 2015;40(10):2368-2378.

37. Zheng LS, Kaneko N, Sawamoto K. Minocycline treatment ameliorates interferon-alpha- induced neurogenic defects and depression-like behaviors in mice. Front Cell Neurosci. 2015;9:5.

38. Mattei D, Djodari-Irani A, Hadar R, et al. Minocycline rescues decrease in neurogenesis, increase in microglia cytokines and deficits in sensorimotor gating in an animal model of schizophrenia. Brain Behav Immun. 2014;38:175-184.

39. Kohman RA, Bhattacharya TK, Kilby C, Bucko P, Rhodes JS. Effects of minocycline on spatial learning, hippocampal neurogenesis and microglia in aged and adult mice. Behav Brain Res. 2013;242:17-24.

40. Kim YK, Na KS, Myint AM, Leonard BE. The role of pro-inflammatory cytokines in neuroinflammation, neurogenesis and the neuroendocrine system in major depression. Prog Neuropsychopharmacol Biol Psychiatry. 2016;64:277-284.

41. Shetty GA, Hattiangady B, Upadhya D, et al. Chronic Oxidative Stress, Mitochondrial Dysfunction, Nrf2 Activation and Inflammation in the Hippocampus Accompany Heightened Systemic Inflammation and Oxidative Stress in an Animal Model of Gulf War Illness. Front Mol Neurosci. 2017;10:182.

42. Butterick TA, Trembley JH, Hocum Stone LL, Muller CJ, Rudquist RR, Bach RR. Gulf War Illnessassociated increases in blood levels of interleukin 6 and C-reactive protein: biomarker evidence of inflammation. BMC Res Notes. 2019;12(1):816.

43. Johnson GJ, Slater BC, Leis LA, Rector TS, Bach RR. Blood Biomarkers of Chronic Inflammation in Gulf War Illness. PLoS One. 2016;11(6):e0157855.

44. Shetty AK, Attaluri S, Kodali M, et al. Monosodium luminol reinstates redox homeostasis, improves cognition, mood and neurogenesis, and alleviates neuro- and systemic inflammation in a model of Gulf War Illness. Redox Biol. 2020;28:101389.

45. Kim HS, Suh YH. Minocycline and neurodegenerative diseases. Behav Brain Res. 2009;196(2):168179.

46. Molina-Torres G, Rodriguez-Arrastia M, Roman P, Sanchez-Labraca N, Cardona D. Stress and the gut microbiota-brain axis. Behav Pharmacol. 2019;30(2 and 3-Spec Issue):187-200.

47. Dubois $T$, Reynaert $C$, Jacques $D$, Lepiece $B$, Zdanowicz N. Role of gut microbiota in the interaction between immunity and psychiatry: a literature review. Psychiatr Danub. 2019;31(Suppl 3):381-385. 
48. Benakis C, Brea D, Caballero S, et al. Commensal microbiota affects ischemic stroke outcome by regulating intestinal gammadelta T cells. Nat Med. 2016;22(5):516-523.

49. Alhasson F, Das S, Seth R, et al. Altered gut microbiome in a mouse model of Gulf War Illness causes neuroinflammation and intestinal injury via leaky gut and TLR4 activation. PLOS One. 2017;12(3):e0172914.

\section{Figures}

A

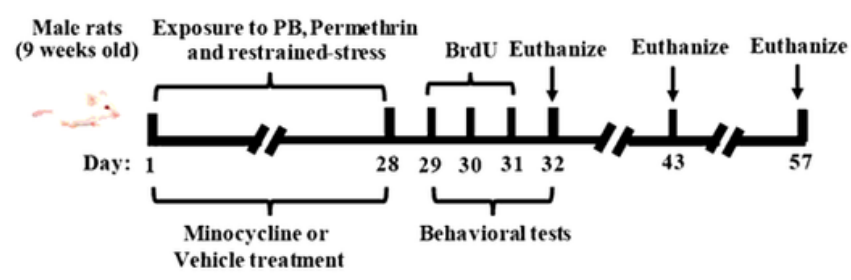

D

Elevated plus maze test

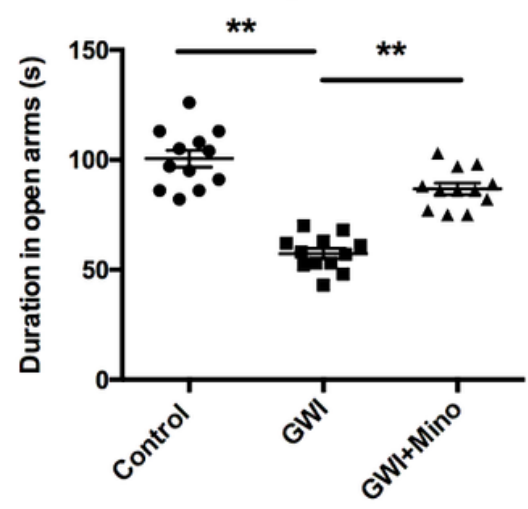

G Novel object recognition test

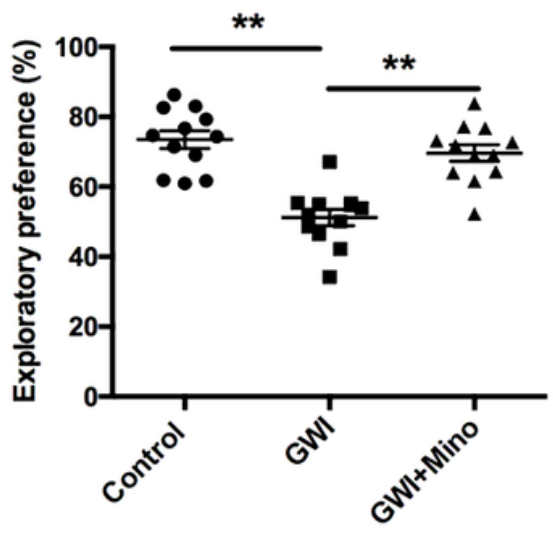

B

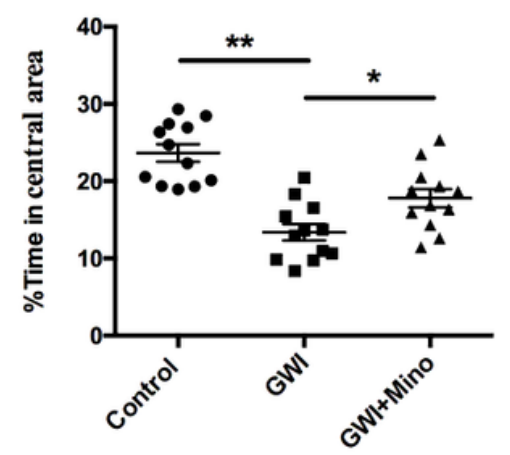

$\mathbf{E}$

Elevated plus maze test

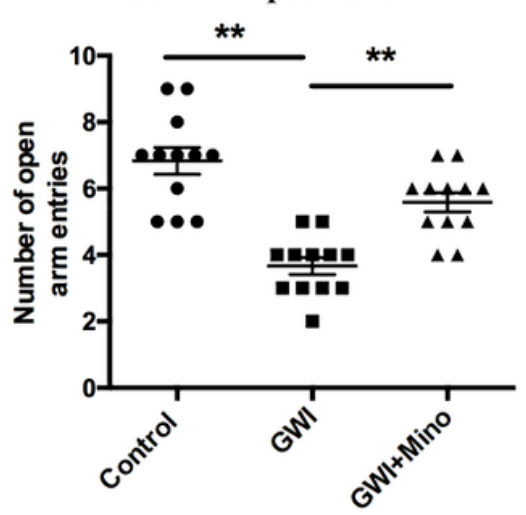

H

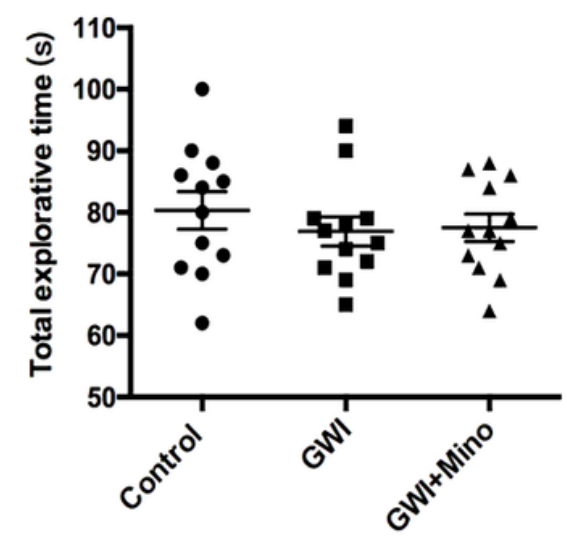

C Open field test

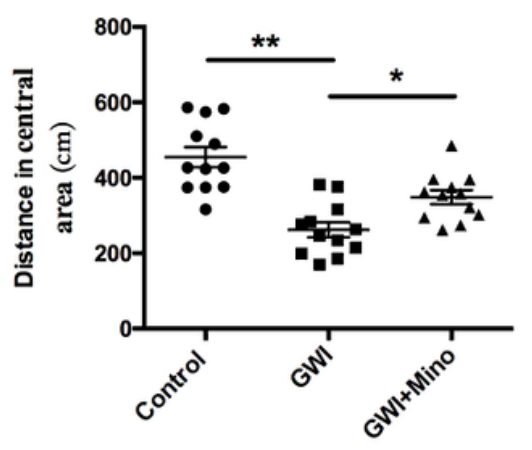

F

Forced swim test

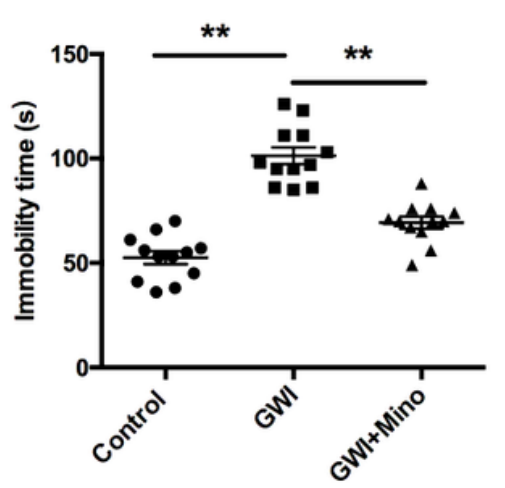

Novel object recognition test

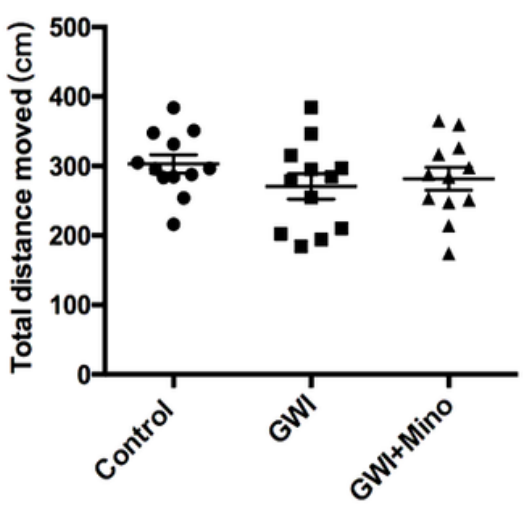


Figure 1

Minocycline treatment alleviated novel object recognition memory and mood function deficits in Gulf War Illness (GWI) rats. (A) Schematic of the experimental design. (B, C) Time spent in the center and distance traversed in the center arena were measured in the open field test among the three groups $(n=12)$. (D, E) Behavior of the three groups of rats in the elevated plus-maze test. Rats in GWI group displayed less time and number of entries in open arms, and treatment with minocycline reversed these effects $(n=12)$. (F) Rats in GWI group displayed more immobility time on the forced swim test, and treatment with minocycline reversed this effect $(n=12)$. (G) Rats in GWI group displayed a lower exploratory preference compared to control rats, and the treatment of minocycline reversed this effect $(n=12)$. $(H, I)$ There were no significant differences between three groups in the total explorative time and the total moved distance $(n=12) .{ }^{*} P<<0.01$, one-way ANOVA and Tukey's post hoc test $(B-I)$.

A

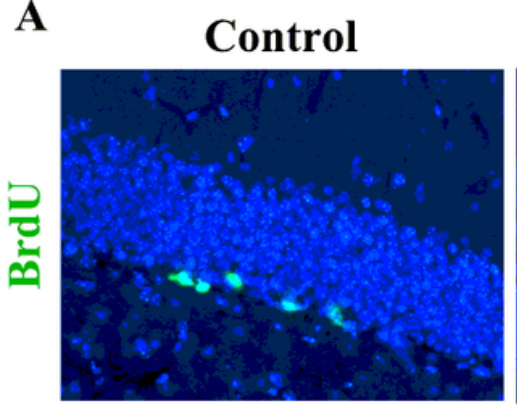

C

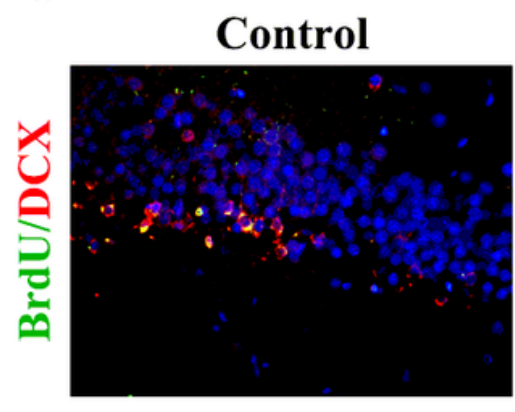

$\mathbf{F}$

Control

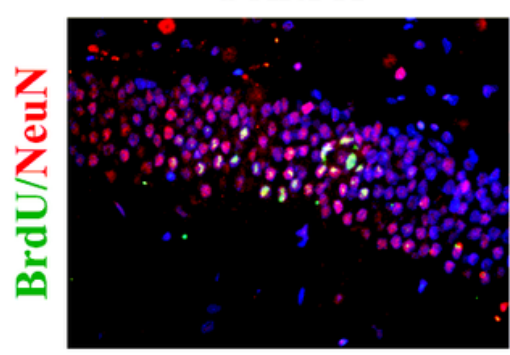

GWI

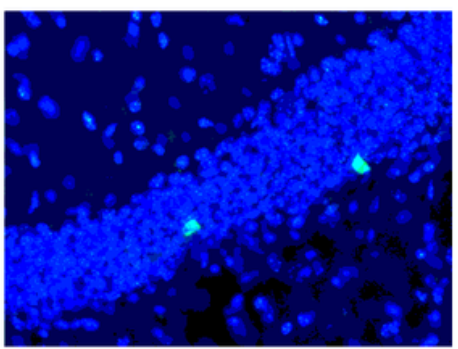

GWI
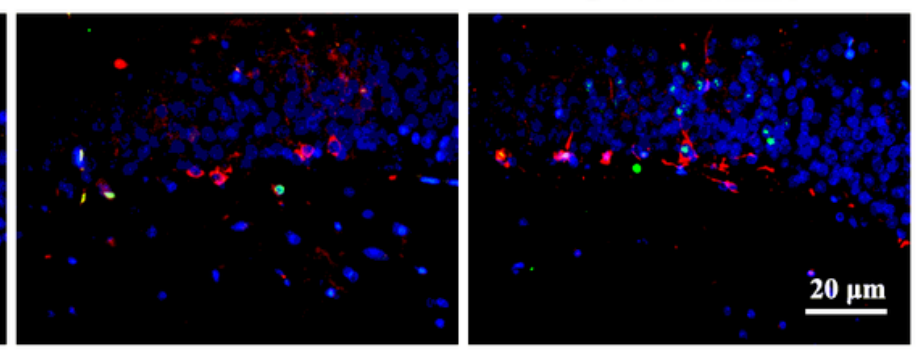

GWI

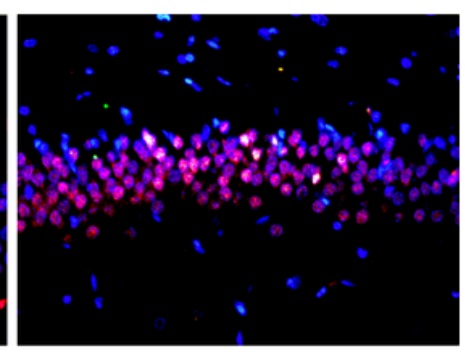

GWI+Mino

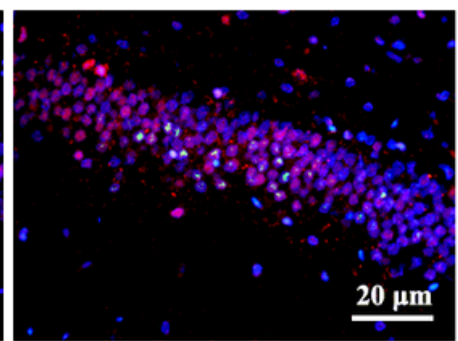

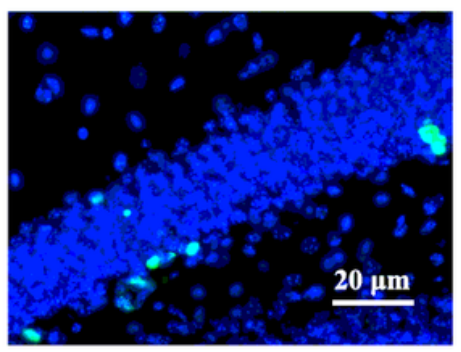

D
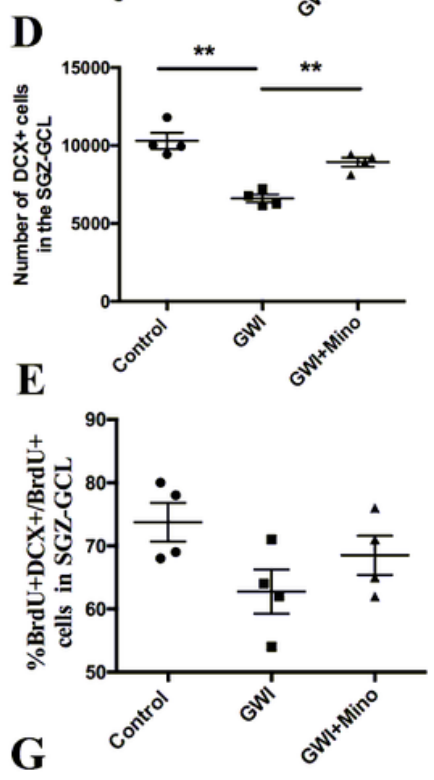

B
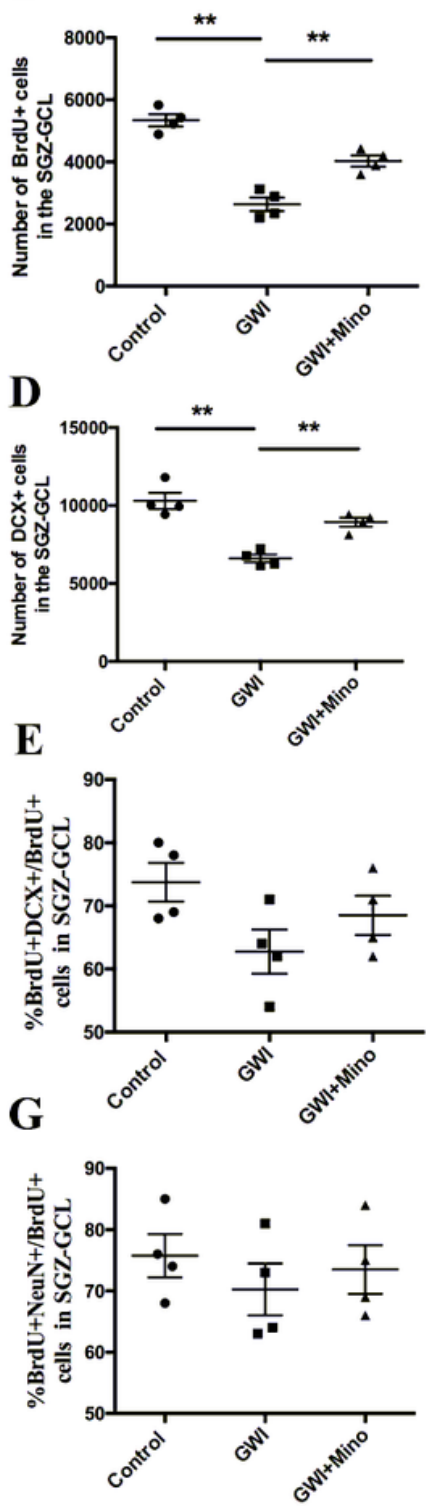

Figure 2 
Minocycline treatment enhanced hippocampal neurogenesis in rats with Gulf War Illness (GWI). (A, B) BrdU-positive cells in the subgranular zone-granule cell layer (SGZ-GCL) for each of the three groups. Rats were sacrificed $24 \mathrm{~h}$ after the last BrdU injection ( $n=4)$. (C-E) BrdU-positive cells and DCX-positive cells in the SGZ-GCL. Mice were sacrificed 14 days after the last BrdU injection. Treated with minocycline rescued the decrease of DCX-positive cells in rats with GWI. But there were no significant differences between three groups in the percentage of BrdU-positive cells expressing DCX $(n=4)$. (F-G) There were no significant differences between three groups in the percentages of BrdU-positive newly born cells that differentiate into NeuN-positive neurons. Rats were sacrificed 28 days after the last BrdU injection $(n=4)$. **P $<0.01$, one-way ANOVA and Tukey's post hoc test $(B, D, E, G)$.

A

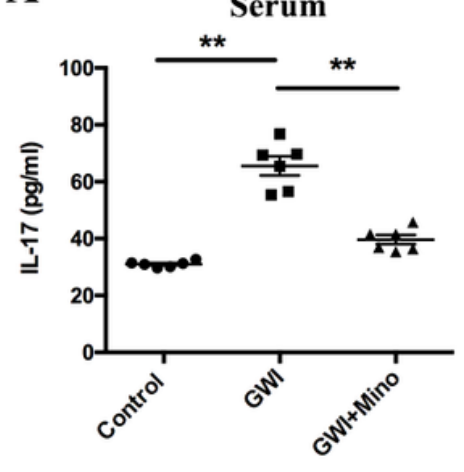

D

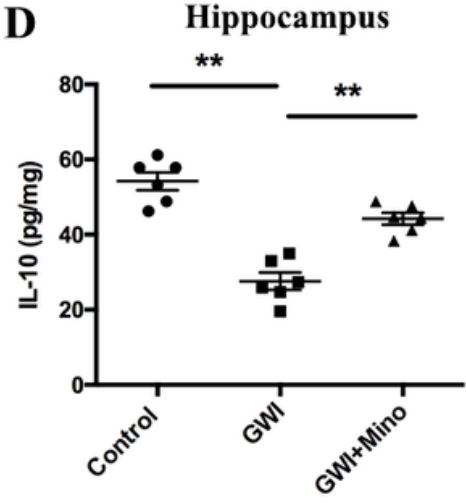

G

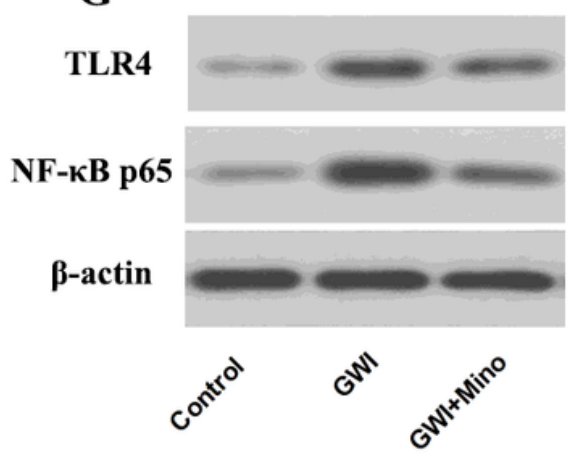

B
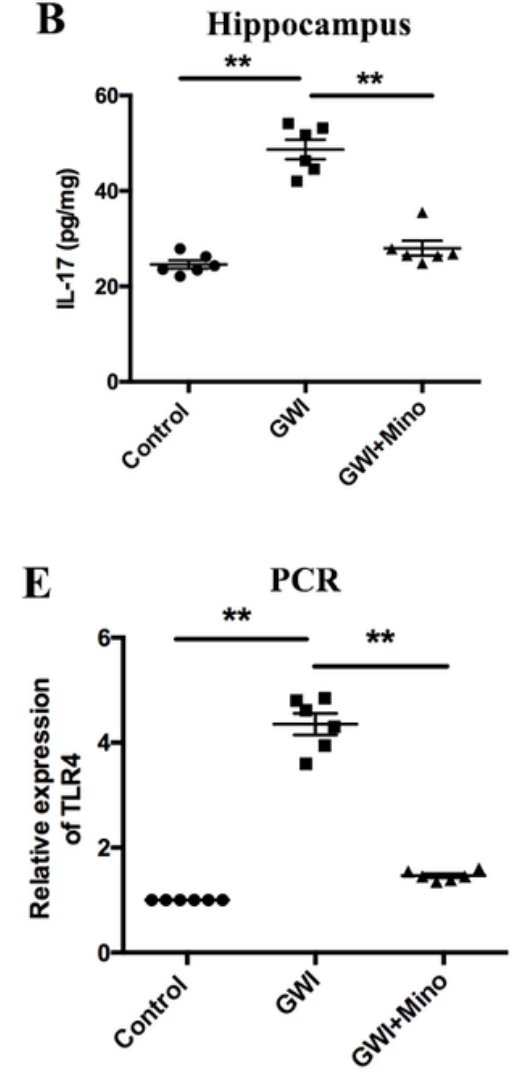

H

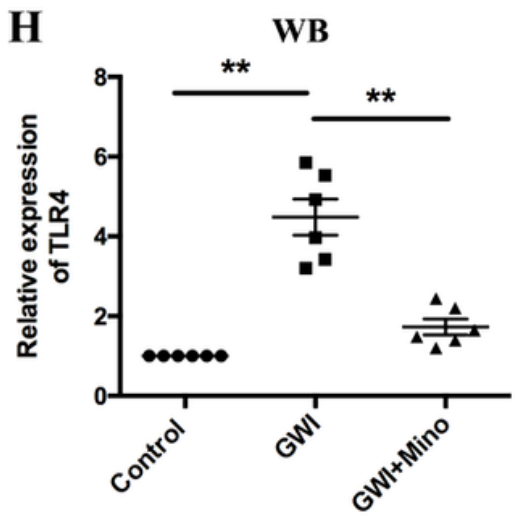

C

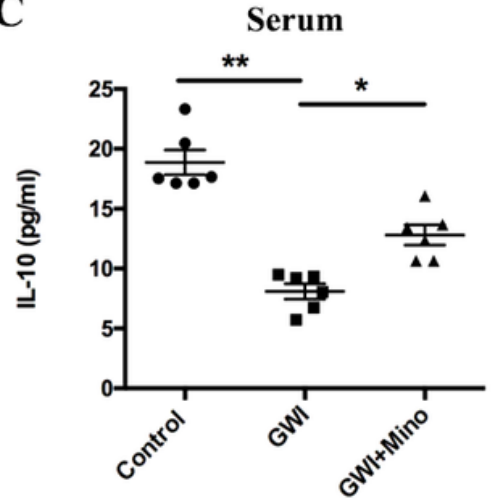

$\mathbf{F}$

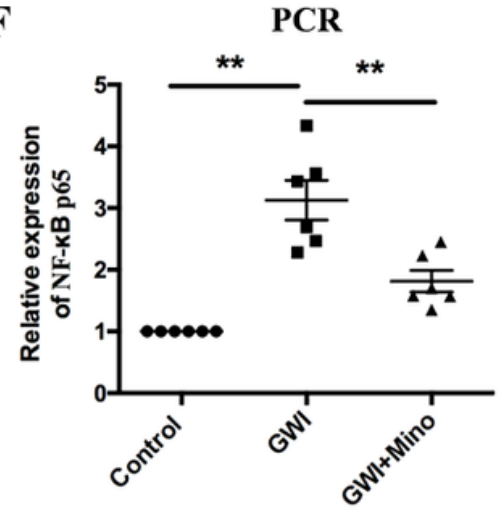

I

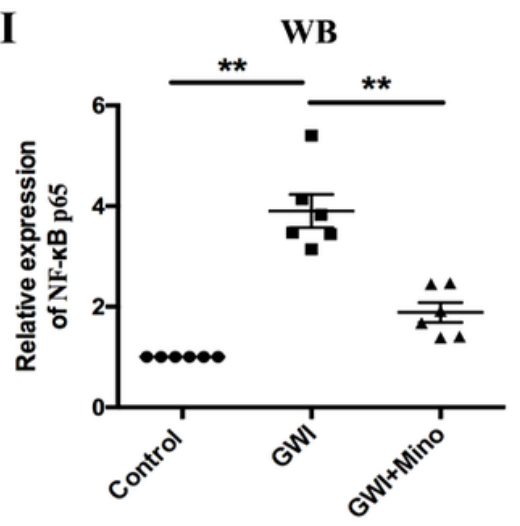

Figure 3

Minocycline treatment attenuated chronic systemic and brain inflammation in rats with Gulf War Illness (GWI). (A-D) Treatment with minocycline decreased the pro-inflammatory markers IL-17 both in serum 
and hippocampus, and decreased the level of anti-inflammatory markers significantly IL-10 both in serum and hippocampus $(n=6)$. (E-I) The expression of TLR4 and NF-KB p65 in hippocampus of different groups rats were detected by PCR and western blotting $(n=6)$. ${ }^{*} P<0.05$, ${ }^{\star *} P<0.01$, one-way ANOVA and Tukey's post hoc test $(A-F, H-I)$.

A

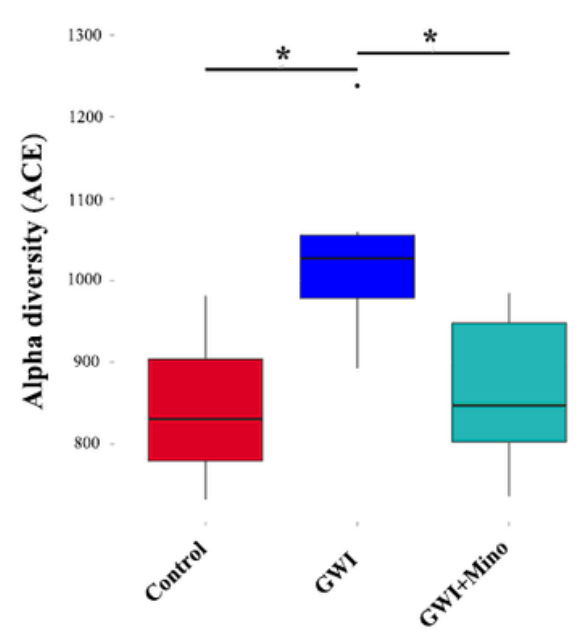

B

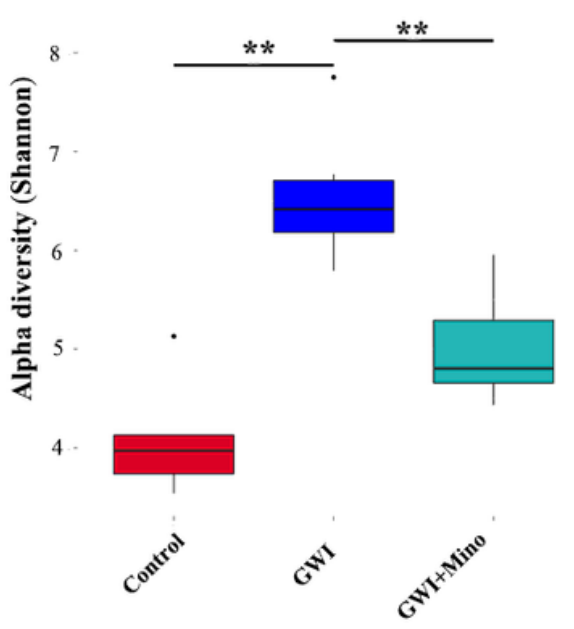

C

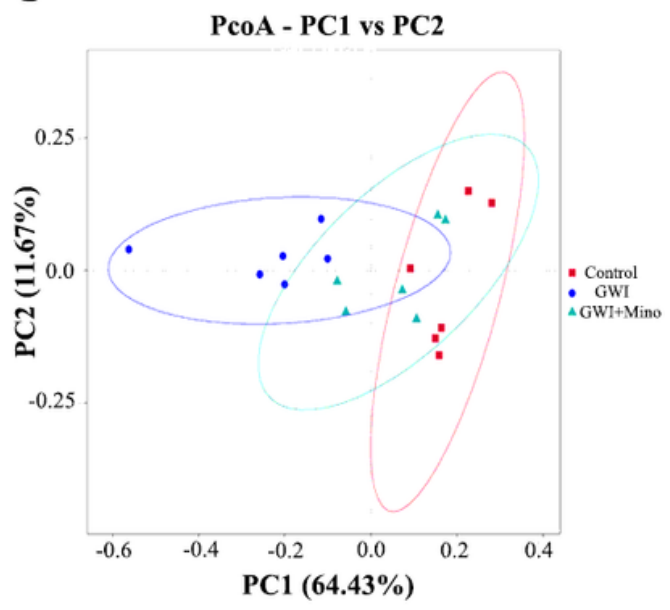

D

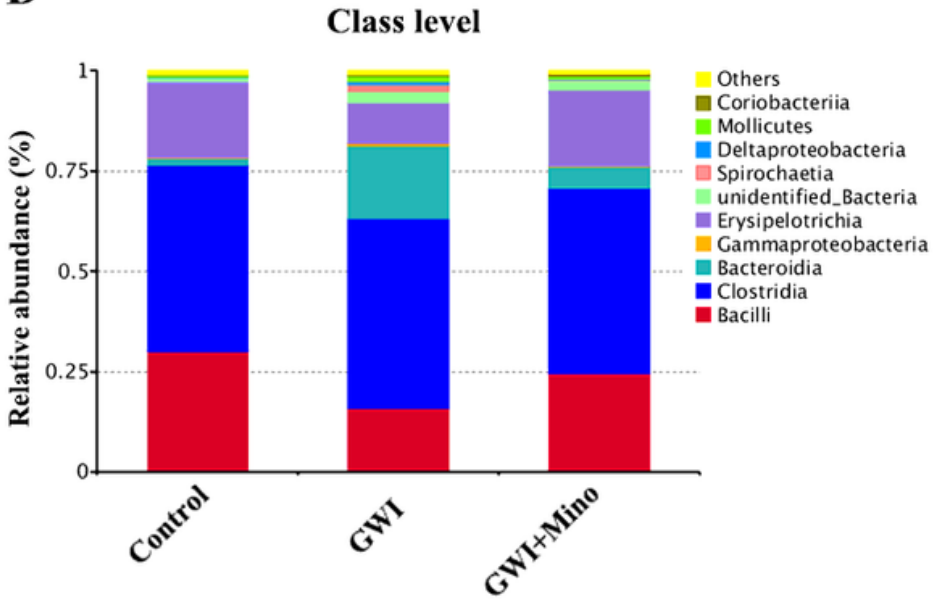

$\mathbf{E}$

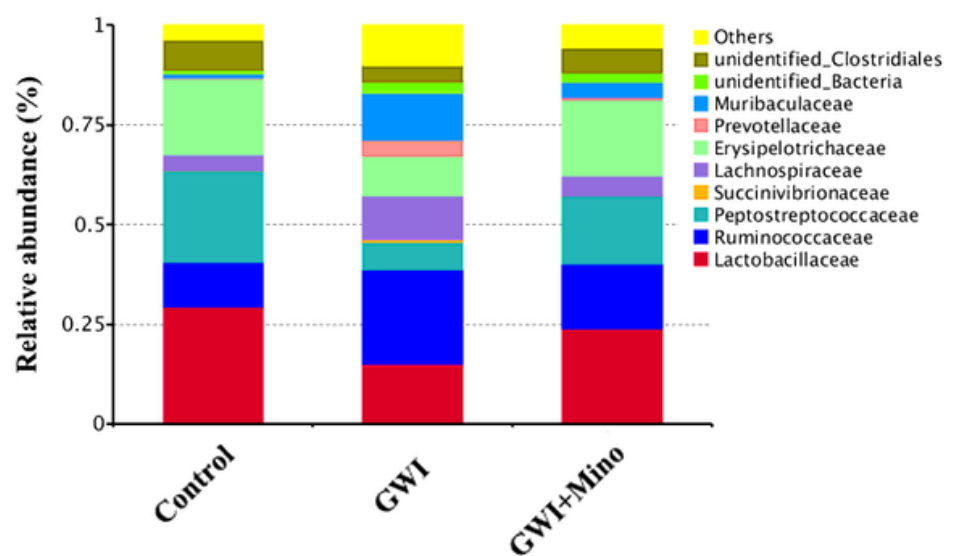

Figure 4

Minocycline treatment rescued gut dysbiosis in rats with Gulf War Illness (GWI). (A, B) The microbial alpha diversity differences between three groups (ACE and Shannon diversity). There were observed significant increase in ACE and Shannon diversity of GWI over Control group, while minocycline treatment rescued these effects significantly $(n=6)$. (C) The PCoA plot showing the microbial composition among three groups based on the weighted UniFrac distances $(n=6)$. (D, E) The relative abundance of specific bacteria at the class level and family level for rats in different groups $(n=6) .{ }^{*} P<0.05,{ }^{*} P<0.01$. Data were analyzed by Kruskal-Wallis test with Tukey's post hoc test $(A, B)$. 